\title{
Ca-Stimulated Type 8 Adenylyl Cyclase Is Required for Rapid Acquisition of Novel Spatial Information and for Working/ Episodic-Like Memory
}

\author{
Ming Zhang, ${ }^{1,2 \star}$ Changjong Moon, ${ }^{1,27 *}$ Guy C.-K. Chan, ${ }^{6}$ Lan Yang, ${ }^{2}$ Fei Zheng,,${ }^{1,3}$ Alana C. Conti, ${ }^{5}$ Lisa Muglia, ${ }^{5}$ \\ Louis J. Muglia, ${ }^{5}$ Daniel R. Storm, ${ }^{6}$ and Hongbing Wang ${ }^{1,2,4}$ \\ ${ }^{1}$ Department of Physiology, ${ }^{2}$ Neuroscience Program, ${ }^{3}$ Department of Biochemistry, and ${ }^{4}$ Cell and Molecular Biology Program, Michigan State University, \\ East Lansing, Michigan 48824, ${ }^{5}$ Department of Pediatrics, Washington University, St. Louis, Missouri 63110, ${ }^{6}$ Department of Pharmacology, University of \\ Washington, Seattle, Washington 98195, and 'Department of Veterinary Anatomy, College of Veterinary Medicine, Chonnam National University, Gwangju \\ 500-757, South Korea
}

Ca-stimulated adenylyl cyclases (ACs) transduce neuronal stimulation-evoked increase in calcium to the production of cAMP, which impinges on the regulation of many aspects of neuronal function. Type 1 and type $8 \mathrm{AC}$ (AC1 and AC8) are the only ACs that are directly stimulated by $\mathrm{Ca}$. Although AC1 function was implicated in regulating reference spatial memory, the function of AC8 in memory formation is not known. Because of the different biochemical properties of AC1 and AC8, these two enzymes may have distinct functions. For example, AC1 activity is regulated by both $\mathrm{Ca}$ and G-proteins. In contrast, AC8 is a pure Ca sensor. It is neither stimulated by $\mathrm{G}_{\mathrm{s}}$ nor inhibited by $\mathrm{G}_{\mathrm{i}}$. Recent studies also suggested that AC1 and AC8 were differentially concentrated at different subcellular domains, implicating that Ca-stimulated signaling might be compartmentalized. In this study, we used AC8 knock-out (K0) mice and found behavioral deficits in memory retention for temporal dissociative passive avoidance and object recognition memory. When examined by Morris water maze, AC8 K0 mice showed normal reference memory. However, the acquisition of newer spatial information was defective in AC8 K0 mice. Furthermore, AC8 KO mice were severely impaired in hippocampus-dependent episodic-like memory when examined by the delayed matching-to-place task. Because AC8 is preferentially localized at the presynaptic active zone, our results suggest a novel role of presynaptic cAMP signaling in memory acquisition and retention, as well as distinct mechanisms underlying reference and working/episodic-like memory.

Key words: adenylyl cyclase; cAMP; synaptic plasticity; learning; memory; knock-out mice

\section{Introduction}

The adaptation of animal behavior to the changing environment requires activity-dependent modification of neuronal functions. Cellular and molecular studies have suggested the role of cAMPand cAMP-dependent protein kinase (PKA)-mediated signal transduction in regulating many forms of neuroplasticity, including long-term potentiation (LTP), long-term depression (LTD), and long-term memory formation (LTM) (Nguyen and Woo, 2003; Wang and Storm, 2003).

Ca-stimulated adenylyl cyclases (ACs) couple the activityevoked $\mathrm{Ca}$ rise to the production of cAMP, which may lead to additional activation of PKA and extracellular signal-regulated kinase (ERK) signaling (Impey et al., 1998). Among all cloned

Received March 6, 2007; revised April 1, 2008; accepted April 1, 2008.

This work was supported by National Institutes of Health Grants MH076906 (H.W.), NS20498 (D.R.S.), MH073601 (D.R.S.), and AG18876 (L.J.M.).

${ }^{*}$ M.Z. and C.M. contributed equally to this work.

Correspondence should be addressed to either of the following: Hongbing Wang, Department of Physiology, Michigan State University, East Lansing, Ml 48824, E-mail: wangho@msu.edu; or Daniel R. Storm, Department of Pharmacology, University of Washington, Seattle, WA 98195, E-mail: dstorm@u.washington.edu. D0I:10.1523/JNEUROSCI.1177-08.2008

Copyright $\odot 2008$ Society for Neuroscience $\quad$ 0270-6474/08/284736-09\$15.00/0
ACs, type 1 and type $8 \mathrm{AC}(\mathrm{AC} 1$ and $\mathrm{AC} 8)$ are stimulated by calcium and calmodulin. Indeed, biochemical and genetic evidence indicated that $\mathrm{AC} 1$ and AC8 are the only ACs that are directly stimulated by Ca in the CNS (Wong et al., 1999). Membrane preparation from the brains of $\mathrm{AC1} / \mathrm{AC} 8$ double knockout (DKO) mice showed no Ca stimulation in AC activity. Interestingly, the late-phase of LTP (L-LTP) in the CA1 region of the hippocampus was significantly impaired in $\mathrm{DKO}$ mice, indicating an essential role of Ca-stimulated AC in synaptic plasticity (Wong et al., 1999). Although DKO mice showed normal shortterm memory for passive avoidance (PA) (Stubley-Weatherly et al., 1996) and contextual memory (Kim and Fanselow, 1992; Logue et al., 1997), their LTM for these two hippocampusdependent tasks was defective (Wong et al., 1999).

Although AC1 and AC8 are both stimulated by Ca/calmodulin, they show different biochemical properties. AC1 is regulated by both G-protein-coupled receptors and Ca (Choi et al., 1992). It is stimulated by $\mathrm{G}_{\mathrm{s}}$ (Wayman et al., 1994) and inhibited by $\mathrm{G}_{\mathrm{i}}$ (Nielsen et al., 1996). In contrast, AC8 is a pure Ca sensor and not regulated by either $\mathrm{G}_{\mathrm{s}}$ or $\mathrm{G}_{\mathrm{i}}$ (Nielsen et al., 1996). Interestingly, AC1 and AC8 may be differentially targeted to distinct subcellular domains, suggesting possible compartmentalization of $\mathrm{Ca}$ - 
stimulated signaling. When the epitope-tagged AC1 and AC8 were overexpressed in hippocampal neurons, we found that AC8, but not AC1, showed concentrated expression pattern at the excitatory synapses (Wang et al., 2003). Moreover, we found that the endogenous AC1 was mainly localized at the postsynaptic density (PSD). The endogenous AC8 was preferentially concentrated at the presynaptic active zone (PAZ) (Conti et al., 2007). These findings suggest that AC1 and AC8 may play distinct roles in regulating activity-dependent plasticity.

When examined by hippocampus-dependent learning paradigms, AC1 knock-out (KO) mice showed impaired reference memory in Morris water maze test (Wu et al., 1995) and normal performance in passive avoidance and contextual fear conditioning. However, the function of AC8 in memory formation is essentially unknown. Interestingly, previous investigation on AC8 $\mathrm{KO}$ mice demonstrated that $\mathrm{AC} 8$ is not required for hippocampus-dependent associative memories, such as passive avoidance and contextual fear conditioning (Wong et al., 1999). In this study, we identified undiscovered roles of AC8, the pure Ca sensor, in memory retention, acquisition of newer spatial information, and working/episodic-like memory.

\section{Materials and Methods}

Animals. The mice mutants for AC8 were generated by gene-specific recombination as described previously (Schaefer et al., 2000). The mice were bred into C57BL/6 background for at least 10 generations. Animals were housed in the university laboratory animal research facility, and all the manipulations were in compliance with the guidelines of Institutional Animal Care and Use Committee at Michigan State University. The mice had ad libitum access to water and food and were housed under $12 \mathrm{~h}$ dark/light cycles.

Behavioral analysis. Open-field analysis was used to measure the activity of wild-type (WT) and AC8 KO mice in a novel environment. Parameters, including total movement time, moving distance, and velocity, were determined by the TruScan Photo Beam Activity System (Coulbourn Instruments, Whitehall, PA).

Passive avoidance. During training, mouse was introduced to the lit half of the training chamber (Coulbourn Instruments) and allowed to explore the lit chamber for $1 \mathrm{~min}$ before opening the trap door. The trap door was closed, and a mild foot shock $(0.7 \mathrm{~mA}$ for $2 \mathrm{~s})$ was delivered immediately after the mouse entered the darkened half. The trained mouse was retained in the dark chamber for $20 \mathrm{~s}$ after the shock and then returned to its home cage. When tested, the trained mouse was reintroduced to the lit chamber. The time spent in the lit half before entering the darkened half was scored as crossover latency and used as index for memory formation. We chose $300 \mathrm{~s}$ as the cutoff value for crossover latency. Mice were removed manually from the lit chamber when the cutoff value was reached.

Temporal dissociative passive avoidance. The behavioral protocol was similar to that of passive avoidance except for that the shock was delivered $10 \mathrm{~min}$ after the mouse entered the dark chamber. The animals were trained by one trial per day for $5 \mathrm{~d}$. The animals were tested on day 6 without shock. During each training trial, the crossover latency was recorded. If the mice reached the cutoff value (300 s) before the fifth trial, they would not be subjected to additional training, and the value of their crossover latency was used for the later sessions. To test long-term memory retention, the trained mouse, which reached the cutoff value (300 s), was tested again $8 \mathrm{~d}$ later. For example, if a trained mouse showed $300 \mathrm{~s}$ crossover latency on day 3 , no more training trial was performed on this particular mouse. The value of $300 \mathrm{~s}$ was used for days 3-6. The mouse was tested for long-term memory retention $8 \mathrm{~d}$ later, which is day 11 .

Object recognition memory. First, the mouse was habituated for $12 \mathrm{~h}$ in the training/testing chamber $(46.5 \times 25 \times 15 \mathrm{~cm}$, length $\times$ width $\times$ height). During training, two objects with different shapes were presented to the mouse for $10 \mathrm{~min}$. One hour after training, another set of objects (one old object and one novel object) was presented to the trained mouse. The interaction of mouse with each object, including approaches and sniffing, was scored. If the mouse had memory retention for the old object, it would show preference to the novel object during testing. The percentage of preference is defined as "number of interaction for a specific object" divided by the "total number of interaction for both objects."

Morris water maze was used for testing hippocampus-dependent spatial memory (Morris et al., 1982). Animal activity was measured by a video-based tracking system (WaterMaze; Coulbourn Instruments). The pool was filled with opaque water (by adding washable white paint) and surrounded by extramaze cues. The escape platform (10 $\mathrm{cm}$ in diameter) was placed in the center of a designated quadrant with its top positioned $1 \mathrm{~cm}$ below the water surface. During the visible platform training, the platform was marked by a flag. Mice were trained by six trials per day for $2 \mathrm{~d}$. The six trials were divided into two blocks with an interval of $1 \mathrm{~h}$ (interblock interval). There were three trials for each block with $10 \mathrm{~min}$ interval between the trials [intertrial interval (ITI)]. Mice were allowed to navigate in the circular pool for up to $60 \mathrm{~s}$ until they found the platform. Mice were allowed to stay on the platform for $30 \mathrm{~s}$. If mice failed to find and land on the platform within $60 \mathrm{~s}$, they were manually guided to the platform. The visible platform was randomly placed in different locations for each trial. The time each mouse spent to land on the platform was scored as escape latency.

After the visible platform training, the mice were further trained by the hidden platform paradigm, during which the platform was placed $1 \mathrm{~cm}$ beneath the opaque water. Mice were trained by four trials (with $1 \mathrm{~h} \mathrm{ITI)}$ per day for $5 \mathrm{~d}$. For each trial, mice were dropped into the pool randomly from four different designated start points.

Probe trials were performed $1 \mathrm{~d}$ after the hidden platform training. With the escape platform removed, the mice were allowed to swim in the pool for $60 \mathrm{~s}$. The time spent in each quadrant, number of crossing for the location of the hidden platform, and swimming speed were recorded.

During the reversal platform training, the hidden platform was moved to the opposite quadrant. The mice were trained by four trials per day (with ITI of $1 \mathrm{~h}$ ) for $4 \mathrm{~d}$. A probe trial was performed $1 \mathrm{~d}$ after the last training session.

The hippocampus-dependent delayed matching-to-place (DMP) task was performed, as described previously (Chen et al., 2000; Zeng et al., 2001), to examine the working/episodic-like memory. Naive WT and AC8 KO mice were first pretrained by the visible platform paradigm (four trials per day for $2 \mathrm{~d}$ with an ITI of $30 \mathrm{~min}$ ). After the pretraining, these mice were trained to find the six new different locations of the hidden platform. For each trial, a maximal duration of $90 \mathrm{~s}$ was allowed for the mice to find the hidden platform. For each platform location, the mice were trained for a maximum of eight trials per day for $2 \mathrm{~d}$ (with an ITI of $10 \mathrm{~min}$ ). If the mice find the platform within $20 \mathrm{~s}$ for three consecutive trials, they will be trained for the next new platform location. The least number of trials was five for each platform location even if a mouse reached the criterion in fewer than five trials.

Hippocampal lesion. Bilateral hippocampal lesions were performed as described previously (de Hoz et al., 2005; Martel et al., 2007). Briefly, mice were anesthetized with pentobarbital sodium (100 mg/kg, i.p.), and mounted on a stereotaxic frame (model 963-LS; David Kopf Instruments, Tujunga, CA) with bregma and lambda on the same horizontal plane. The injection cannulas were placed to the dorsal hippocampus with coordinates of $2.0 \mathrm{~mm}$ posterior, $1.4 \mathrm{~mm}$ lateral, and $1.6 \mathrm{~mm}$ ventral. Ibotenic acid $(0.3 \mu \mathrm{l}$ with a concentration of $10 \mathrm{mg} / \mathrm{ml}$ in PBS) was delivered at a flow rate of $0.05 \mu \mathrm{l} / \mathrm{min}$ by a WPI (Sarasota, FL) syringe pump. After injection, the infusion cannula was kept in place for $2 \mathrm{~min}$ and then pulled out slowly. Sham lesions were performed the same way but injected with $0.3 \mu \mathrm{l}$ of PBS. At the end of behavioral test, animals were perfused with ice-cold PBS. The brains were removed, fixed in $6 \%$ paraformaldehyde/PBS, and cryoprotected with $30 \%$ sucrose/PBS. Coronal sections (30 $\mu \mathrm{m}$ thick) were subjected to histological examination by cresyl violet staining.

Detection of endogenous $A C 1$ and AC8. The forebrain tissues including hippocampus were freshly dissected from WT, AC1 KO, and AC8 KO mice. After homogenization, the synaptosomes were isolated by sucrose gradient centrifugation $(100,000 \times g$ for $1.5 \mathrm{~h})$ as described previously (Phillips et al., 2001; Conti et al., 2007). The purified synaptosomes were 
further separated into extrasynaptic, PSD, and PAZ fractions as described previously (Conti et al., 2007) and subjected to $4-12 \%$ SDSPAGE. AC1 and AC8 were detected by Western blot analysis with the ECL methods (SuperSignal WestDura; Pierce, Rockford, IL). Antibodies against AC1 (1:500; rabbit polyclonal; developed in house), AC8 (1:500; rabbit polyclonal; Santa Cruz Biotechnology, Santa Cruz, CA), PSD-95 (1:4000; monoclonal; Affinity BioReagents, Golden, CO), and Rab3A (1:1000; rabbit polyclonal; Affinity BioReagents) were used.

Data analysis. Two-way repeated-measures ANOVA was performed for water maze and temporal dissociative passive avoidance (TDPA) data (genotype and time/trial as between/within-subject factor). Three-way repeated-measures ANOVA was used for delayed matching-to-pace task. Student's $t$ test was used to assess significance for data between two groups. Data were expressed as the mean \pm SEM. Differences with $p$ values $<0.05$ were considered significant.

\section{Results}

AC8 KO mice showed normal locomotor activity, passive avoidance memory, and shock sensitivity

We first examined the basal locomotor activity of AC8 KO mice in a novel environment by the open-field analysis. WT mice and AC8 KO mice showed comparable movement time, similar travel distance, and ambulatory velocity (supplemental Fig. 1, available at www.jneurosci.org as supplemental material). These data indicated normal movement of AC8 KO mice.

It was reported that $\mathrm{AC} 1 / \mathrm{AC} 8 \mathrm{DKO}$ mice were significantly impaired in two hippocampus-dependent tasks, passive avoidance memory and contextual memory (Wong et al., 1999). However, single KO mice for AC8 showed normal memory retention in both tasks (Wong et al., 1999). These results indicated that AC8 activity is not necessary to support certain forms of associative memory. Here, we confirmed that the memory retention is normal with AC8 KO mice for passive avoidance (supplemental Fig. 2, available at www.jneurosci.org as supplemental material). Both WT and AC8 KO mice showed significant increase in crossover latency when tested $24 \mathrm{~h}$ after training $\left(F_{(1,14)}=548 ; p<\right.$ 0.001 , two-way ANOVA), and there was no significant difference between the genotypes $\left(F_{(1,14)}=650 ; p=0.4\right)$. We also found no difference in shock sensitivity between WT mice and AC8 KO mice. WT mice and AC8 KO mice showed similar reaction during the delivery of the mild electric foot shock $(0.7 \mathrm{~mA}$ for $2 \mathrm{~s})$. They showed similar increase in ambulatory velocity during the shock and an after-shock reduction in movement (supplemental Fig. 3, available at www.jneurosci.org as supplemental material).

\section{AC8 KO mice are impaired for temporal dissociative passive avoidance}

Although AC8 KO mice were normal for contextual and passive avoidance memory, we reasoned that it might be attributable to the intrinsic features of these two paradigms. These two paradigms are very strong and less demanding training protocols and may not be sensitive enough to detect behavioral phenotypes in AC8 KO mice. We used a modified passive avoidance protocol, TDPA, in which the delivery of an aversive unconditioned stimulus (US) (the mild electric foot shock) was delayed. In the standard passive avoidance, the mild electric foot shock was delivered immediately after the mouse entered the darkened half of the training chamber. In the TDPA, the foot shock was delivered 10 min after mouse entering the darkened half, so that the association of the conditioned stimuli (CSs) with the unconditioned stimuli was weaker. Normally a single passive avoidance training may lead to very strong memory formation, as indicated by reaching the cutoff value for crossover latency during testing (supplemental Fig. 2, available at www.jneurosci.org as supple-

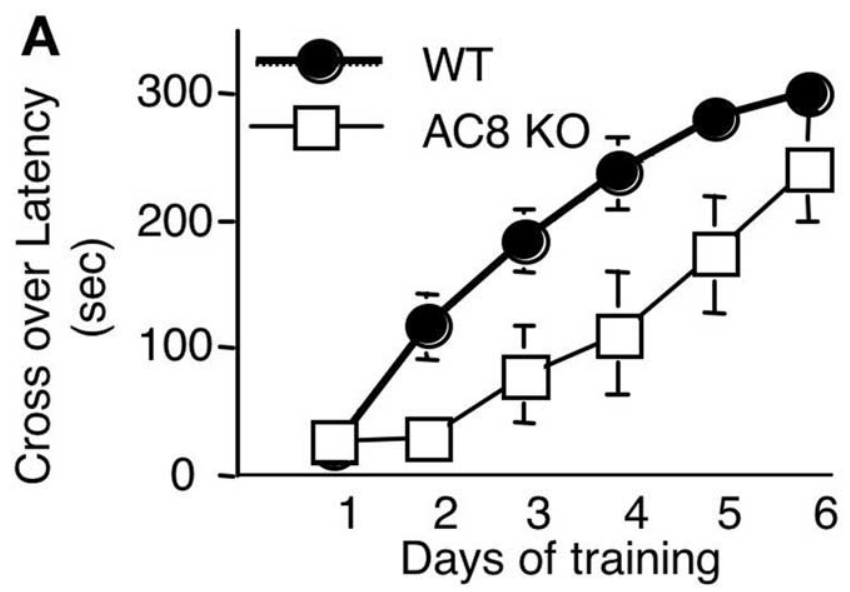

B

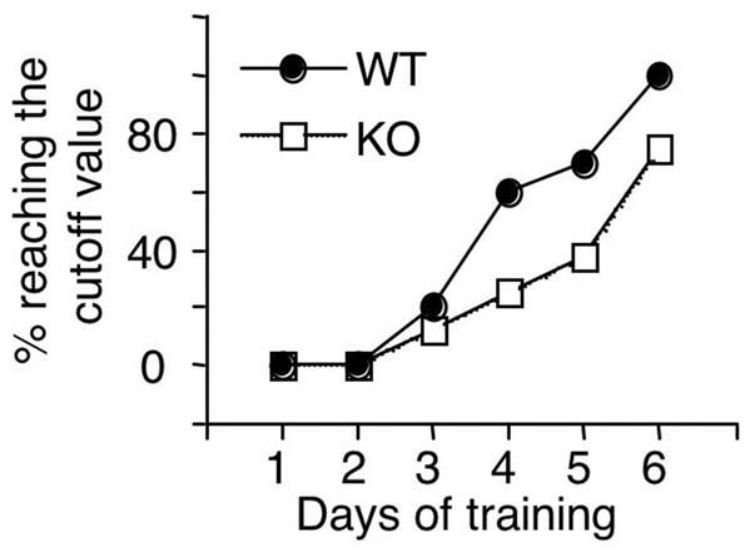

Figure 1. AC8 KO mice show deficits in TDPA. WT $(n=10)$ and AC8 KO $(n=8)$ mice, at the age of 2-3 months, were trained by TDPA everyday (from day 1 to day 5 ). The trained mice were tested $24 \mathrm{~h}$ after each training (from day 2 to day 6 ). The crossover latency was recorded. $\boldsymbol{A}, \mathrm{AC} 8$ KO mice showed slower learning than WT mice, as indicated by the lower crossover latency on testing days $2-5$. All values are average \pm SEM. $\boldsymbol{B}$, The percentage of mice reaching the cutoff value was lower for AC8 $\mathrm{KO}$ mice.

mental material). When trained by the TDPA, multiple training sessions are needed for mice to reach the cutoff value (Fig. 1A).

Compared with WT mice, AC8 KO mice showed significantly slower increase in crossover latency when trained for the TDPA. Although the two-way ANOVA analysis demonstrated significant learning and memory formation for both WT and AC8 KO mice $\left(F_{(5,80)}=42 ; p<0.001\right)$, significant difference in crossover latency was revealed between WT and AC8 KO mice $\left(F_{(1,16)}=13\right.$; $p=0.002)$. The trial $\times$ genotype interaction also revealed significant difference $\left(F_{(5,80)}=2.7 ; p=0.03\right)$. These data demonstrated a slower learning curve with AC8 $\mathrm{KO}$ mice in TDPA. In addition, WT mice required less training sessions to reach the cutoff value (300 s of crossover latency). The percentage of animals with $300 \mathrm{~s}$ crossover latency was higher for WT mice in all testing sessions (Fig. $1 \mathrm{~B}$ ). After five consecutive daily trainings, all WT mice reached $300 \mathrm{~s}$ crossover latency when tested on day 6 . In contrast, $75 \%$ of AC8 KO mice ( six of eight) reached the cutoff value on day 6 .

To test long-term memory retention, the mice reached the $300 \mathrm{~s}$ cutoff value after TDPA training were tested again $8 \mathrm{~d}$ later. Because not all AC8 KO mice showed cutoff value on day 6, we only chose the individuals (six of eight) with $300 \mathrm{~s}$ crossover latency for the 8 day memory retention test. We observed signif- 




C

B

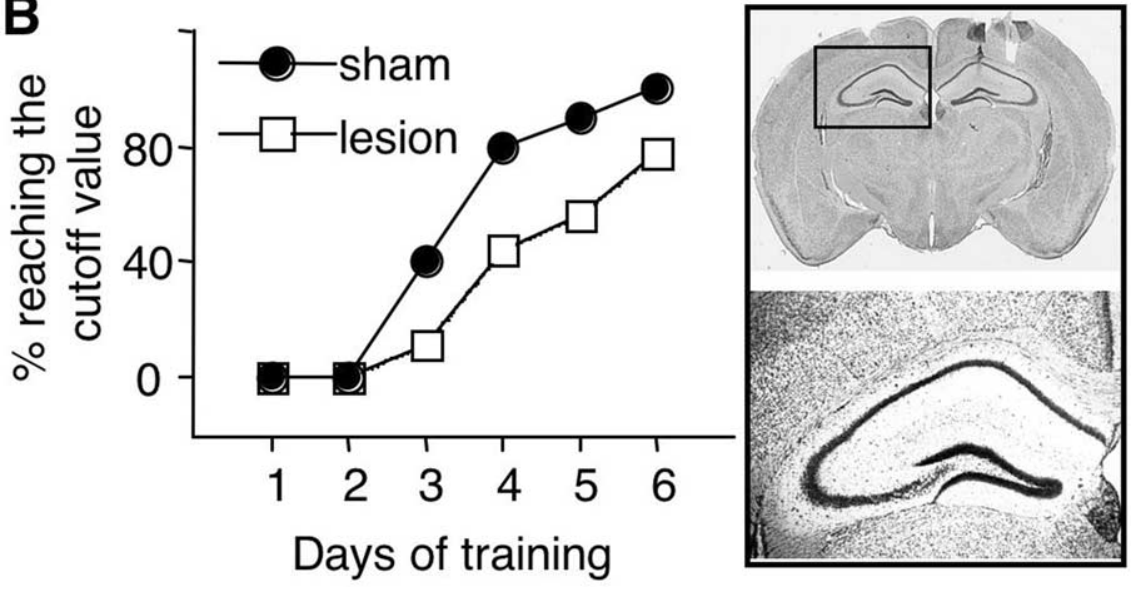

Figure 2. Memory formation for TDPA depends on hippocampus. Mice with hippocampal lesion (injected with ibotenic acid, $n=9$ ) and sham-operated mice (injected with PBS, $n=10$ ) were trained by TDPA as described in Figure 1. $\boldsymbol{A}$, Lesioned mice displayed poor TDPA performance, as indicated by shorter crossover latency during testing. $\boldsymbol{B}$, The percentage of animals reaching the cutoff value (300 s) was lower for lesioned mice. C, D, The hippocampal morphology was examined by cresyl violet staining. Hippocampal lesion occurred in ibotenic acid-injected $(\boldsymbol{C})$ but not in PBS-injected mice $(\boldsymbol{D})$. The boxed regions in the top panels were enlarged and shown in the bottom panels.

icant loss of TDPA memory for AC8 KO mice. The crossover latency of WT was $275 \pm 13 \mathrm{~s}$ when tested $8 \mathrm{~d}$ later. In contrast, the value for AC8 KO mice was $157 \pm 52 \mathrm{~s}$ ( $p=0.05$, Student's $t$ test). The difference between WT and AC8 KO mice during training and testing was not attributable to different shock sensitivity (supplemental Fig. 3, available at www.jneurosci.org as supplemental material).

To determine whether TDPA depends on the function of hippocampus, we did bilateral hippocampal lesions. After lesion/ surgery, mice were recovered for $9 \mathrm{~d}$ and tested for TDPA. As shown in Figure 2, both groups significantly increased their crossing-over latency during training $\left(F_{(5,85)}=54.4 ; p<0.0001\right)$. However, the lesioned mice (injected with ibotenic acid) displayed significant slower learning than the sham-operated mice (injected with vehicle PBS) $\left(F_{(1,17)}=10.7 ; p<0.01\right.$, repeatedmeasures two-way ANOVA). Post hoc comparison showed that there was significant difference between the lesioned and the sham group on day $2(p=0.01)$ and day $3(p=0.04)$ and a tendency to significant difference on day $4(p=0.06)$. Furthermore, the percentage of animals with 300 s crossover latency (the cutoff value) was lower for the lesioned mice in all testing sessions (Fig. 2B). After five consecutive daily trainings, all sham- operated mice reached $300 \mathrm{~s}$ crossover latency when tested on day 6. In contrast, $78 \%$ of the lesioned mice (seven of nine) reached the cutoff value on day 6 . Histology assessment revealed that significant hippocampal lesions occurred in ibotenic acid-injected mice (Fig. 2C). No apparent hippocampal damages were observed in the sham-operated mice (Fig. 2D). These results implicated that TDPA depends on intact hippocampus.

\section{AC8 KO mice are impaired for object recognition memory}

We further examined AC8 KO mice by another sensitive hippocampus-dependent paradigm, object recognition memory (Myhrer, 1988; Reed and Squire, 1997). Because there is no reinforcement (such as an aversive unconditioned stimulus) during the object recognition training, a single training trial usually results in weaker memory formation, which may last for $<1$ d. During the $10 \mathrm{~min}$ training session, WT and AC8 KO mice showed similar interaction with the objects $(38 \pm 3$ for WT mice, $37 \pm 3$ for AC8 KO mice; $p>0.05$, Student's $t$ test), indicating normal motivation and exploratory activity. WT and AC8 $\mathrm{KO}$ mice also displayed equal preference to the two objects during training (Fig. $3 A$ ). During testing, one conditioned old object was replaced by a novel object. If mice retained memory for the old objects, they would show preference to the novel object. When tested $1 \mathrm{~h}$ after training, only WT mice, but not AC8 $\mathrm{KO}$ mice, showed significant preference to the novel object $(67 \pm 2 \%$ for WT mice, $52 \pm 2 \%$ for AC8 KO mice; $p<0.001$, Student's $t$ test) (Fig. $3 B$ ). These data indicated that AC8 is required for the formation of recognition memory.

\section{The function of AC8 in spatial reference memory}

We examined hippocampus-dependent spatial memory by Morris water maze (Morris et al., 1982). We first trained mice with the visible platform paradigm, in which the animals learn to find the escape platform with an attached visual cue. Although WT showed better improvement for the first four trials on day 1 , there was no significant difference in escape latency after 12 trials ( $p=$ 0.267, two-way ANOVA) (Fig. 4A). Both genotypes showed significant improvement in escape latency $\left(F_{(11,187)}=19.5 ; p<\right.$ 0.001, two-way ANOVA). Animals of both group showed similar swimming speed during visible platform training $(1.8 \pm 0.1$ arbitrary units for both genotypes; $p=0.317, t$ test). These data indicated that $\mathrm{AC} 8 \mathrm{KO}$ mice were normal in motor activity, vision, and motivation to escape from the water.

We further examined AC8 KO mice with the hidden platform paradigm, in which animals learned to find the escape platform by using the extramaze cues. AC8 KO mice showed similar performance to that of WT mice. Both group displayed significant training-related decrease in escape latency, correlating well with the number of trials (Fig. $4 B)\left(F_{(19,323)}=5 ; p<0.001\right.$, two-way 

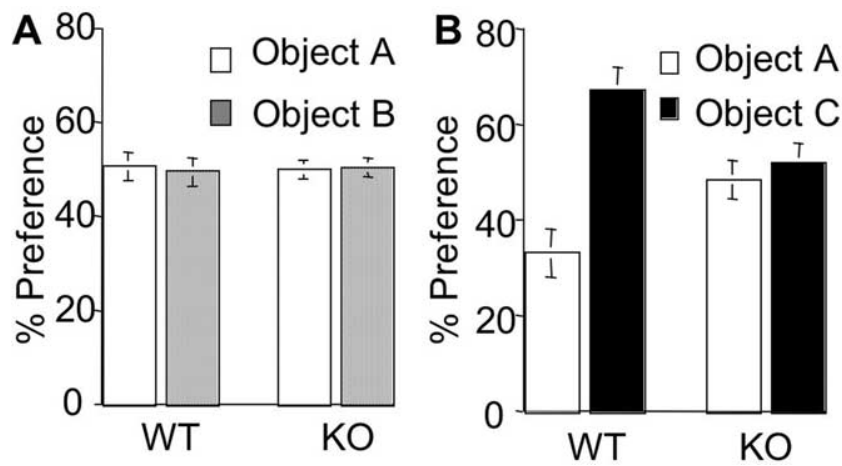

Figure 3. AC8 KO mice show deficits in object recognition memory. WT $(n=9)$ and AC8 KO $(n=8)$ mice were trained for object recognition memory. $A$, WT and AC8 KO mice showed equal preference to the two objects during training. $\boldsymbol{B}$, WT, but not AC8 KO mice, showed significant preference to the novel object during testing. The values are average \pm SEM.

ANOVA), and there was no significant difference between WT and AC8 KO mice $\left(F_{(1,17)}=201 ; p=0.919\right.$, two-way ANOVA). During the probe test, both WT and AC8 KO mice spent significant more time in the target quadrant ( $46 \pm 4 \%$ for WT mice, $49 \pm 5 \%$ for AC8 KO mice; $p=0.674$, Student's $t$ test) (Fig. $4 C$ ). The number of crossing the platform location was also comparable between the two groups (WT and AC8 KO mice) $(3.4 \pm 0.5$ for WT mice, $2.9 \pm 1$ for AC8 KO mice; $p=0.63$, Student's $t$ test). During the probe test, AC8 KO mice also displayed similar swim speed to that of WT mice (data not shown). These data indicated that AC8 is not required for reference spatial memory.

\section{The function of AC8 in working/episodic-like memory during the delayed matching-to-place task}

We next tested whether AC8 KO mice could relearn new platform formation. During the reversal trials, the platform was moved to the opposite quadrant from the previously trained position. After $4 \mathrm{~d}$ of training, both WT and AC8 KO mice significantly improved in escape latency $\left(F_{(15,255)}=5.4 ; p<0.001\right.$, two-way ANOVA) (Fig. 5A). There was no statistical difference in escape latency between WT and AC8 KO mice for the whole training session $\left(F_{(1,17)}=1.4 ; p=0.253\right.$, two-way ANOVA). Furthermore, AC8 KO and WT mice showed comparable percentage of time in the target quadrant (Fig. $5 B)(45 \pm 3 \%$ for WT mice, $49 \pm$ $4 \%$ for AC8 KO mice; $p=0.317, t$ test), number of crossing for the location of the escape platform $(3.1 \pm 0.5$ for WT mice, $4.0 \pm$ 0.7 for AC8 KO mice; $p=0.205, t$ test), and swim speed ( $1.8 \pm 0.1$ for WT mice, $1.8 \pm 0.1$ for AC $8 \mathrm{KO}$ mice; $p=0.465, t$ test) during the probe trial after the last reversal training.

Although there was no significant difference between WT and AC8 KO mice in escape latency, the $p$ value for the interaction between genotype and trial was $<0.05\left(F_{(15,255)}=2.2 ; p=0.008\right.$, two-way ANOVA). During the first day of reversal training, we noticed that the reduction of escape latency was significantly less for AC8 KO mice in the third and fourth trials (Fig. 5A). The post hoc comparison detected difference for trials 3 and $4\left(F_{(1,17)}=7\right.$, $p=0.018$ for trial $3 ; F_{(1,17)}=8, p=0.013$ for trial 4$)$ and suggested that $\mathrm{AC} 8 \mathrm{KO}$ mice might be impaired for acquiring newer spatial information. Therefore, we further examined memory acquisition by moving the escape platform to another location. The platform was moved clockwise to two new locations (as indicated in Fig. $5 C$ ) on 2 consecutive days. AC8 KO mice showed significant impairments in finding the new platform location on day 2 $\left(F_{(1,17)}=7 ; p=0.017\right.$, two-way ANOVA) (Fig. $\left.5 C\right)$.

The data from Figure 5 suggested that AC8 might be required for working/episodic-like memory. Therefore, we examined naive cohorts of WT and AC8 KO mice by DMP task in Morris water maze (Chen et al., 2000; Zeng et al., 2001). During the DMP task, which requires dynamic acquisition of ongoing events and measures hippocampus-dependent working/episodic-like memory, mice were trained to learn the six changing locations of the platform. For each platform location, the mice ware trained by a maximum of eight trials per day for up to $2 \mathrm{~d}$. If the mice were able to find the platform within $20 \mathrm{~s}$ for three consecutive trials, they would be trained for the next platform position. However, a minimum of five trials was performed for each platform location. Again, WT and AC8 KO mice showed comparable performance during the visible platform pretraining (data not shown). They also displayed similar swimming speed during all pretraining and DMP training sessions (data not shown). Although both WT and AC8 KO mice showed learning and improvement in escape latency for all platform locations $\left(F_{(5,55)}=6.9, p<0.0001\right.$, for WT mice; $F_{(5,60)}=6.6, p<0.0001$ for AC8 KO mice; two-way ANOVA), WT mice showed significantly faster learning of the new platform location than AC8 KO mice (Fig. $6 A, B)\left(F_{(1,23)}=\right.$ 33.7; $p<0.0001$, three-way repeated-measures ANOVA). The difference in trial $\times$ genotype interaction was also significant $\left(F_{(4,92)}=7.6 ; p<0.0001\right)$. To determine the function of AC8 in rapid learning after a single trial for the new platform, we calculated the "saving time," which was the reduction of escape latency between the first and second trials. AC8 $\mathrm{KO}$ mice showed much smaller saving value than WT mice (Fig. 6C). The average saving for the first three platform positions was $36 \pm 6 \mathrm{~s}$ for WT mice and $16 \pm 7 \mathrm{~s}$ for AC8 KO mice (Fig. 6C) $(p<0.05, t$ test). The average saving for the last three platform locations was $47 \pm 4 \mathrm{~s}$ for WT mice and $17 \pm 6 \mathrm{~s}$ for AC8 KO mice (Fig. $6 C)(p<0.001$, $t$ test). We also noticed that AC8 KO mice needed more trials to reach the criterion for each platform location (i.e., $<20 \mathrm{~s}$ in escape latency) (Fig. 6D). For the first three platform locations, the average number of trials was $6.6 \pm 0.4$ for WT mice and $9.4 \pm 0.5$ for AC8 KO mice (Fig. 6D) $(p<0.01, t$ test). For the last three locations, it was $4.8 \pm 0.2$ for WT mice and $7.6 \pm 0.7$ for AC8 KO mice (Fig. $6 D)(p<0.005, t$ test). These data strongly suggested that AC8 is required for the hippocampus-dependent working/ episodic-like memory. When data were analyzed for the last two platform locations, AC8 KO mice displayed more dramatic impairments for the improvement in escape latency (supplemental Fig. $4 A$, available at www.jneurosci.org as supplemental material), saving time (supplemental Fig. $4 B$, available at www.jneurosci.org as supplemental material), and trial numbers needed to reach the escape latency criterion of $20 \mathrm{~s}$ (supplemental Fig. 4C, available at www.jneurosci.org as supplemental material). These data demonstrated that $\mathrm{AC} 8$ is required for working/episodiclike memory.

\section{AC8 is preferentially localized in the presynaptic active zone and copurifies with Rab3A}

As described previously, mice mutants with $\mathrm{AC1}$, another Castimulated AC, showed impairments in spatial reference memory (Wu et al., 1995). In contrast, AC8 KO mice displayed normal reference memory in Morris water maze, as indicated by their normal performance in the probe tests (Figs. 4C, 5B). Possibly, the different phenotypes could be attributable to the distinct biochemical properties and differential subcellular localization of these two Ca-stimulated enzymes. Here, we reconfirmed that $\mathrm{AC} 1$ is concentrated in the PSD fraction and AC8 is concentrated in the PAZ fraction. AC1 was copurified with PSD-95 but not with Rab3A (Fig. $7 A, C, D$ ). Rab3A is a small GTPase, whose func- 

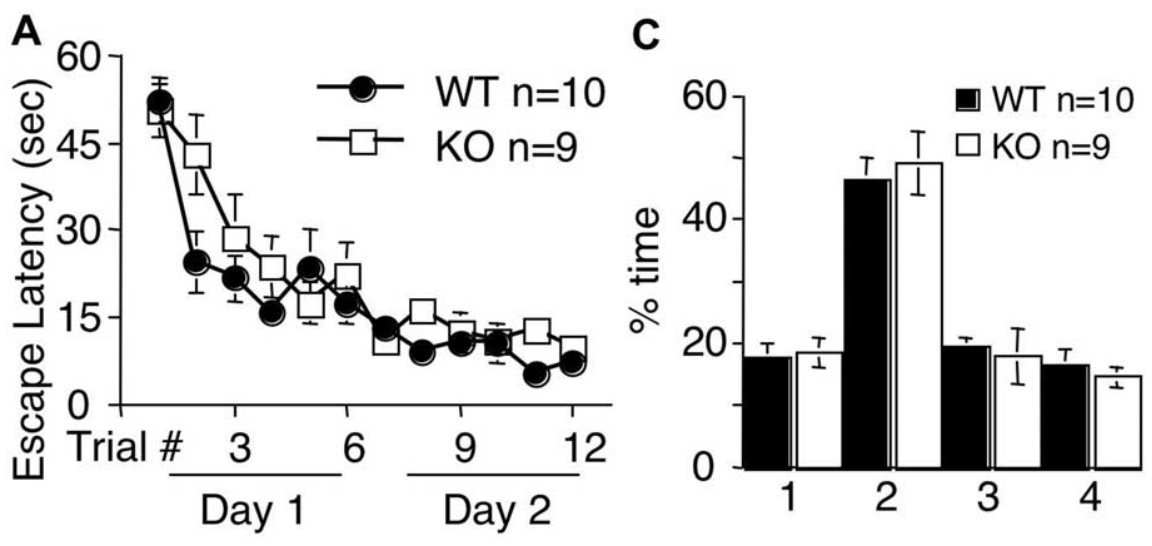

B

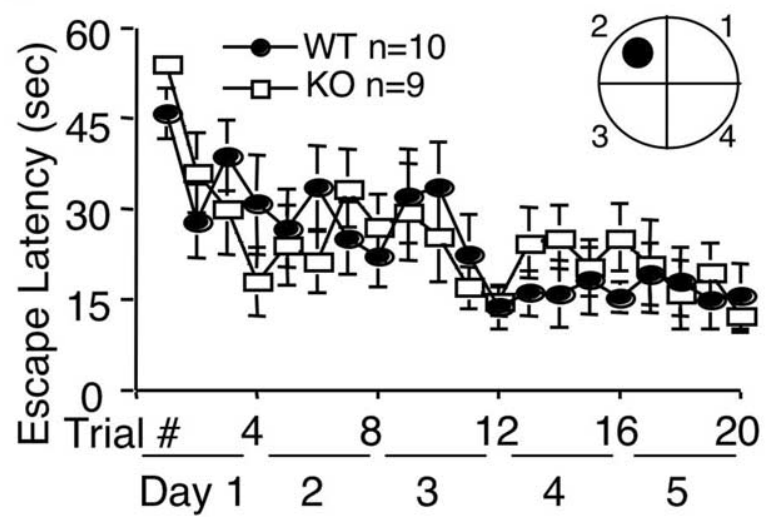

Figure 4. AC8 KO mice show normal reference memory for Morris water maze. $A$, WT and AC $8 \mathrm{KO}$ mice were first trained by the visible platform paradigm and showed comparable improvement in escape latency. $\boldsymbol{B}$, The same set of mice were further trained by the hidden platform paradigm and showed comparable improvement in escape latency. During training, the hidden platform was positioned in the center of a designated quadrant (arbitrarily quadrant 2 as indicated in the inset diagram). C, During the probe trial, both WT and AC8 KO mice spent significantly more time in the target quadrant (quadrant 2). All values are average \pm SEM.

tion is demonstrated in regulating vesicle fusion and neurotransmitter release (Schluter et al., 2002; Sudhof, 2004). Interestingly, Rab3A mutant mice showed similar phenotypes to that of AC8 KO mice. They are both impaired for mossy fiber LTP (mfLTP) (Castillo et al., 1997; Wang et al., 2003) and acquisition of newer spatial information (D'Adamo et al., 2004, and this study). Here, we show that AC8 was also copurified with Rab3A, which was present in the PAZ but not in PSD preparations (Fig. $7 B-D$ ). Although the antibodies against AC1 and AC8 detected a few nonspecific bands (Fig. $7 A, B$ ), they appeared to be highly specific for $\mathrm{AC} 1$ and $\mathrm{AC} 8$. The specific immunosignal was lost in $\mathrm{AC} 1 \mathrm{KO}$ mice when AC1 antibody was used (Conti et al., 2007) (Fig. 7A). Similarly, AC8 antibody failed to detect AC8 in AC8 KO mice (Conti et al., 2007) (Fig. 7B). We consider the doublets (detected by AC8 antibody) in the PSD fraction nonspecific signals, because they were also present in AC8 KO samples (Fig. 7B). These molecules might be extensively enriched in PSD and picked up by nonspecific antibody binding. They were not present in PAZ preparations from AC8 KO mice (Fig. 7B).

\section{Discussion}

$\mathrm{Ca} /$ calmodulin-stimulated ACs couple the two major second messengers, Ca and cAMP, in the CNS. Supported by biochemistry, molecular, and genetic studies, it was concluded that $\mathrm{AC1}$ and AC8 are the only ACs that are directly activated by $\mathrm{Ca}$. The activity-dependent upregulation of the enzymatic activity of AC1 and AC8 may be pivotal for the activation of many signaling molecules, such as PKA, ERK, and cAMPresponsive element binding protein (CREB), which play essential roles in regulating synaptic plasticity (Nguyen and Woo, 2003).

The function of Ca-stimulated ACs in regulating neuroplasticity was addressed by using gene knock-out strategies. DKO for both AC1 and AC8 showed no latephase LTP in the CA1 region of the hippocampus (Wong et al., 1999). When examined by passive avoidance and contextual fear conditioning, the DKO mice showed no LTM retention. These data demonstrated that Ca-stimulated ACs are required for many aspects of plasticity. Recently, it was shown that the activitydependent activation of ERK and CREB was lost in DKO neurons (Sindreu et al., 2007).

The contribution of $\mathrm{AC} 1$ and $\mathrm{AC} 8$ to Ca-stimulated cyclase activity was determined by the use of DKO mice and single $\mathrm{KO}$ mice of AC1 and AC8. The Castimulated AC activity was significantly reduced in the hippocampus of $\mathrm{AC} 1 \mathrm{KO}$ and AC8 KO and totally lost in DKO mice (Wong et al., 1999). Despite the significant reduction in Ca-stimulated $\mathrm{AC}$ activity, AC8 KO mice showed normal transcription-dependent L-LTP at the Schaffer collateral/CA1 synapses (Wong et al., 1999). Although the level of CA1 LTP was lower during the first hour after induction (Wu et al., 1995), L-LTP was normal in AC1 KO mice (Wong et al., 1999). Furthermore, $\mathrm{AC} 1 \mathrm{KO}$ and $\mathrm{AC} 8 \mathrm{KO}$ mice both showed normal LTM for passive avoidance and contextual fear conditioning (Wong et al., 1999). These data indicated that AC1 or AC8 alone might be sufficient to support certain forms of synaptic plasticity.

Are AC1 and AC8 redundant? Although passive avoidance and contextual memory do not depend on AC1 activity, AC1 KO mice showed significant deficits in spatial reference memory formation. In the Morris water maze paradigm, AC1 KO mice showed normal acquisition in the hidden platform training but were impaired in the probe test (Wu et al., 1995). As described previously, the regulatory properties of $\mathrm{AC} 1$ and $\mathrm{AC} 8$ are different. Compared with $\mathrm{AC} 1$, whose activity is regulated by both G-proteins and Ca, AC8 is a pure Ca sensor. Recently, we found that the endogenous $\mathrm{AC} 1$ is preferentially localized in the postsynaptic density, whereas AC8 is detected mainly in the presynaptic active zone (Conti et al., 2007) (Fig. 7). Therefore, they may play distinct roles in synaptic plasticity. Although AC8 KO mice showed normal CA1 LTP, they were impaired in CA1 LTD (Schaefer et al., 2000). Interestingly, AC8 is required for mossy fiber LTP (Wang et al., 2003), which is mechanistically different from CA1 LTP and does not depend on the activation of NMDA receptors (Zalutsky and Nicoll, 1990; Johnston et al., 1992). However, the function of AC8 in memory formation is basically unknown. In this study, we found that AC8 is required for a more sensitive form of passive avoidance memory, TDPA. We assume that TDPA and standard PA are essentially similar. They both 
involve the same US and CS, except that the coupling of US and CS is delayed in TDPA. With this kind of perturbation, more training sessions are required for animals to form strong memory. The same TDPA protocol was successfully used to detect impairments with mice mutants for an $\beta$-amyloid precursor protein interacting protein FE65 (B. Wang et al., 2004). By the hippocampal lesion experiments, we, for the first time, demonstrated that this sensitive paradigm of PA is indeed hippocampus dependent. Compared with WT mice, AC8 KO mice showed weaker memory formation, as well as weaker memory retention in TDPA. Although AC8 KO mice showed normal passive avoidance and contextual memory, they were defective in object recognition memory, which also depends on the function of hippocampus.

We found that the function of $\mathrm{AC} 8$ and $\mathrm{AC} 1$ is different in regulating spatial memory. Whereas the reference memory is significantly impaired in $\mathrm{ACl} \mathrm{KO}$ mice $\mathrm{Wu}$ et al., 1995), AC8 KO mice showed normal performance in acquisition and the probe test for the hidden platform test. However, the ability to acquire newer platform location was lost in AC8 $\mathrm{KO}$ mice (Fig. $5 C$ ). Such impairment suggests a role of AC8 in working/episodic-like memory, which involves dynamic acquisition of ongoing information. Therefore, we further examined AC8 KO mice with DMP task using Morris water maze. Compared with WT mice, AC8 KO mice showed slower learning for the six changing platform locations, as indicated by slower improvement in latency and smaller saving values.

The postsynaptic mechanisms for learning and memory formation were intensively investigated. Recently, emerging evidence started to suggest the role of presynaptic function in certain forms of hippocampus-dependent learning (Powell, 2006). Specifically, the functional relevance of LTP at the mossy fiber/CA3 synapses (mfLTP) in memory formation was investigated in genetically engineered mice. As described previously, the deletion of Rab3A, a molecule involved in neurotransmitter release, resulted in severe impairments of mfLTP (Castillo et al., 1997). However, the Rab3A KO mice showed normal reference memory in the hidden platform test of Morris water maze. Their contextual memory formation was also comparable with that of WT mice (Powell et al., 2004). Although a recent report suggested a role of Rab3A in the regulation of emotion (Yang et al., 2007), the phenotypes of Rab3A KO mice in cued fear conditioning were controversial (Powell et al., 2004; Yang et al., 2007). The lack of behavioral phenotypes was also demonstrated in mice mutant for PKA. Although genetic deletion of the $C \beta 1$ or the RI $\beta$ isoform of PKA caused significant deficits in mfLTP, these KO mice displayed normal hidden platform performance and contextual memory (Huang et al., 1995). Although the functional role of mfLTP in learning was challenged, D'Adamo et al. (2004) reported interesting defective phenotypes in reversal and episodiclike memory with Rab3A KO mice (D'Adamo et al., 2004). Our study revealed a striking similarity between Rab3A KO and AC8 KO mice. They are both defective in mfLTP, show normal contextual memory (Wong et al., 1999; Powell et al., 2004) and hidden platform performance, but display impairments in reversal and DMP test. Although our data do not prove the causal effects of mfLTP, we suggest that this form of presynaptic plasticity may be required for more sensitive and demanding learning paradigms. This possibility was demonstrated by the phenotypes of AC8 KO mice in passive avoidance and TDPA. We showed that AC8 KO mice were normal for the standard passive avoidance but impaired for the more sensitive TDPA test. It would be interesting to examine Rab3A and PKA mutant mice with TDPA and recognition memory, in which $\mathrm{AC} 8 \mathrm{KO}$ mice were also defective.

Although the essential role of Ca-stimulated ACs in memory was well accepted, the function of presynaptic and postsynaptic cAMP signaling is unknown. Because of the different subcellular localization, we suggest that cAMP-regulated memory is mainly mediated by $\mathrm{AC} 1$ at the postsynaptic site and by AC 8 at the presynaptic site. It is important to mention that $\mathrm{ACl} \mathrm{KO}$ mice are also defective for mfLTP (Villacres et al., 1998). Although plasticity at the mossy fiber synapses is considered to be mainly presynaptic (Zalutsky and Nicoll, 1990; Weisskopf and Nicoll, 1995), the role of postsynaptic $\mathrm{Ca}$ and cAMP was demonstrated (Yeckel et al., 1999; J. Wang et al., 2004). Because mild deficits in CA1 LTP were observed with AC1 $\mathrm{KO}$ mice, it is premature to postulate how regulation of mfLTP by cAMP at presynaptic and postsynaptic sites affects reference and working memory. Never- 


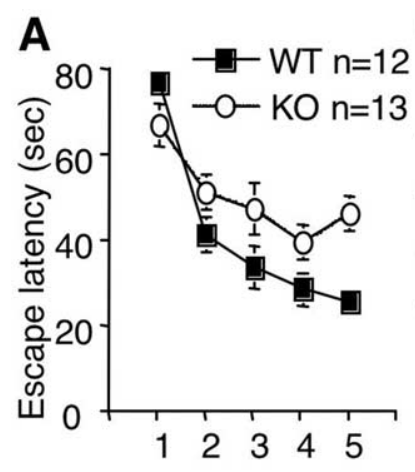

C WT $\mathrm{n}=12 \square \mathrm{KO} \mathrm{n}=13$
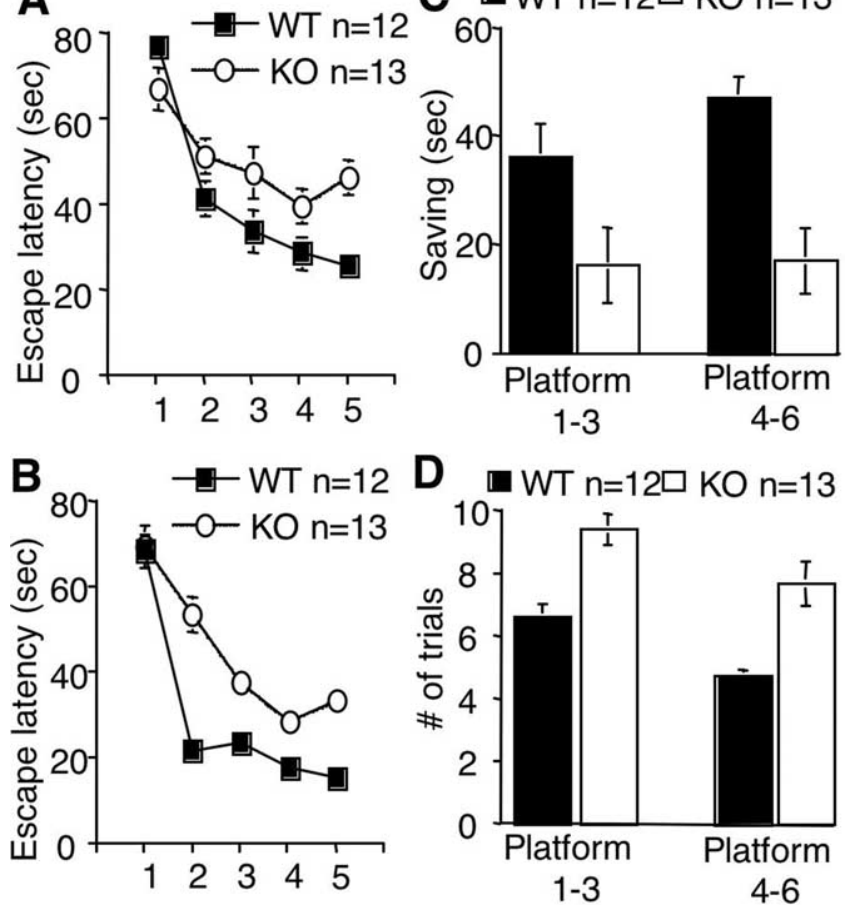

Figure 6. AC8 KO mice show impairments in DMP task. WT and AC8 KO mice were trained in the Morris water maze by the DMP task. The values were averaged for the first three platform locations (platforms 1-3) and the last three platform locations (platforms 4-6). $\boldsymbol{A}$, Escape latency for the first five trials when averaged for the first three platform locations. $\boldsymbol{B}$, Escape latency for the first five trials when averaged for the last three platform locations. $\boldsymbol{C}$, The reduction of escape latency between the first and second trials was calculated and expressed as saving. $\boldsymbol{D}$, The number of trials required for WT or AC $8 \mathrm{KO}$ mice to reach the arbitrary criterion (20 s escape latency) are plotted. The values are average \pm SEM. In $\boldsymbol{C}$ and $\boldsymbol{D}$, the values are presented for the first three (platforms 1-3) and last three (platforms 4-6) platform locations, as indicated.

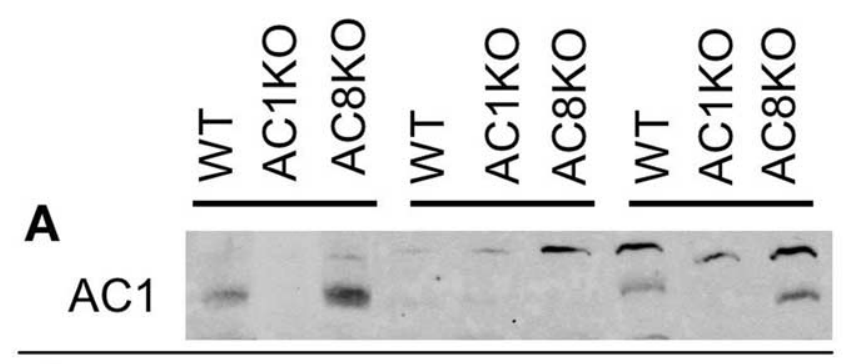

B

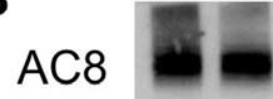

C

PSD95

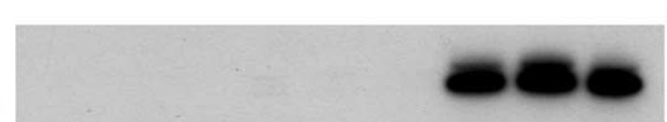

D

Rab3A

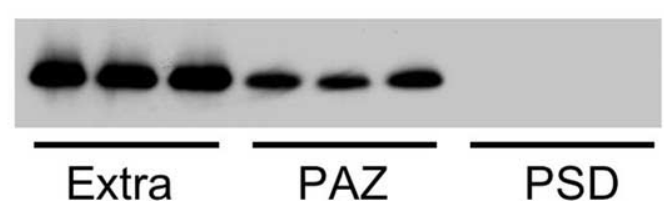

Figure 7. $A C 8$ is copurified with Rab3A in the PAZ. Brain homogenates from WT, AC1 KO, and AC8 KO mice were separated into extrasynaptic, PAZ, and PSD fractions. $A-D$, The presence of AC1 (A), AC8 (B), PSD-95 (C), and Rab3A (D) was detected by Western blot analysis. theless, the distinct properties of $\mathrm{AC} 1$ and $\mathrm{AC} 8$ are reflected by the different phenotypes in $\mathrm{AC} 1 \mathrm{KO}$ and $\mathrm{AC} 8 \mathrm{KO}$ mice.

In summary, we identified novel roles of AC8 in regulating memory retention. Furthermore, we found that AC8 and AC1 have distinct function in spatial memory. Whereas AC1 is required for reference memory, AC8 is required for the acquisition of newer spatial information and working/episodic-like memory. Our results also suggest that compartmentalized Ca-stimulated cAMP signaling with distinct biochemical properties may differentially regulate neuronal function and behavior.

\section{References}

Castillo PE, Janz R, Sudhof TC, Tzounopoulos T, Malenka RC, Nicoll RA (1997) Rab3A is essential for mossy fibre long-term potentiation in the hippocampus. Nature 388:590-593.

Chen G, Chen KS, Knox J, Inglis J, Bernard A, Martin SJ, Justice A, McConlogue L, Games D, Freedman SB, Morris RG (2000) A learning deficit related to age and beta-amyloid plaques in a mouse model of Alzheimer's disease. Nature 408:975-979.

Choi EJ, Wong ST, Hinds TR, Storm DR (1992) Calcium and muscarinic agonist stimulation of type I adenylylcyclase in whole cells. J Biol Chem 267:12440-12442.

Conti AC, Maas JR JW, Muglia LM, Dave BA, Vogt SK, Tran TT, Rayhel EJ, Muglia LJ (2007) Distinct regional and subcellular localization of adenylyl cyclases type 1 and 8 in mouse brain. Neuroscience 146:713-729.

D'Adamo P, Wolfer DP, Kopp C, Tobler I, Toniolo D, Lipp HP (2004) Mice deficient for the synaptic vesicle protein Rab3a show impaired spatial reversal learning and increased explorative activity but none of the behavioral changes shown by mice deficient for the Rab3a regulator Gdil. Eur J Neurosci 19:1895-1905.

de Hoz L, Moser EI, Morris RG (2005) Spatial learning with unilateral and bilateral hippocampal networks. Eur J Neurosci 22:745-754.

Huang YY, Kandel ER, Varshavsky L, Brandon EP, Qi M, Idzerda RL, McKnight GS, Bourtchouladze R (1995) A genetic test of the effects of mutations in PKA on mossy fiber LTP and its relation to spatial and contextual learning. Cell 83:1211-1222.

Impey S, Obrietan K, Wong ST, Poser S, Yano S, Wayman G, Deloulme JC, Chan G, Storm DR (1998) Cross talk between ERK and PKA is required for $\mathrm{Ca}^{2+}$ stimulation of CREB- dependent transcription and ERK nuclear translocation. Neuron 21:869-883.

Johnston D, Williams S, Jaffe D, Gray R (1992) NMDA-receptorindependent long-term potentiation. Annu Rev Physiol 54:489-505.

Kim JJ, Fanselow MS (1992) Modality-specific retrograde amnesia of fear. Science 256:675-677.

Logue SF, Paylor R, Wehner JM (1997) Hippocampal lesions cause learning deficits in inbred mice in the Morris water maze and conditioned-fear task. Behav Neurosci 111:104-113.

Martel G, Blanchard J, Mons N, Gastambide F, Micheau J, Guillou JL (2007) Dynamic interplays between memory systems depend on practice: the hippocampus is not always the first to provide solution. Neuroscience 150:743-753.

Morris RG, Garrud P, Rawlins JN, O’Keefe J (1982) Place navigation impaired in rats with hippocampal lesions. Nature 297:681-683.

Myhrer T (1988) Exploratory behavior and reaction to novelty in rats with hippocampal perforant path systems disrupted. Behav Neurosci 102:356-362.

Nguyen PV, Woo NH (2003) Regulation of hippocampal synaptic plasticity by cyclic AMP-dependent protein kinases. Prog Neurobiol 71:401-437.

Nielsen MD, Chan GC, Poser SW, Storm DR (1996) Differential regulation of type I and type VIII Ca ${ }^{2+}$-stimulated adenylyl cyclases by Gi-coupled receptors in vivo. J Biol Chem 271:33308-33316.

Phillips GR, Huang JK, Wang Y, Tanaka H, Shapiro L, Zhang W, Shan WS, Arndt K, Frank M, Gordon RE, Gawinowicz MA, Zhao Y, Colman DR (2001) The presynaptic particle web: ultrastructure, composition, dissolution, and reconstitution. Neuron 32:63-77.

Powell CM (2006) Gene targeting of presynaptic proteins in synaptic plasticity and memory: across the great divide. Neurobiol Learn Mem 85:2-15.

Powell CM, Schoch S, Monteggia L, Barrot M, Matos MF, Feldmann N, Sudhof TC, Nestler EJ (2004) The presynaptic active zone protein 
RIMlalpha is critical for normal learning and memory. Neuron 42:143-153.

Reed JM, Squire LR (1997) Impaired recognition memory in patients with lesions limited to the hippocampal formation. Behav Neurosci 111:667-675.

Schaefer ML, Wong ST, Wozniak DF, Muglia LM, Liauw JA, Zhuo M, Nardi A, Hartman RE, Vogt SK, Luedke CE, Storm DR, Muglia LJ (2000) Altered stress-induced anxiety in adenylyl cyclase type VIII-deficient mice. J Neurosci 20:4809-4820.

Schluter OM, Khvotchev M, Jahn R, Sudhof TC (2002) Localization versus function of Rab3 proteins. Evidence for a common regulatory role in controlling fusion. J Biol Chem 277:40919-40929.

Sindreu CB, Scheiner ZS, Storm DR (2007) $\mathrm{Ca}^{2+}$-stimulated adenylyl cyclases regulate ERK-dependent activation of MSK1 during fear conditioning. Neuron 53:79-89.

Stubley-Weatherly L, Harding JW, Wright JW (1996) Effects of discrete kainic acid-induced hippocampal lesions on spatial and contextual learning and memory in rats. Brain Res 716:29-38.

Sudhof TC (2004) The synaptic vesicle cycle. Annu Rev Neurosci 27:509-547.

Villacres EC, Wong ST, Chavkin C, Storm DR (1998) Type I adenylyl cyclase mutant mice have impaired mossy fiber long-term potentiation. J Neurosci 18:3186-3194.

Wang B, Hu Q, Hearn MG, Shimizu K, Ware CB, Liggitt DH, Jin LW, Cool BH, Storm DR, Martin GM (2004) Isoform-specific knockout of FE65 leads to impaired learning and memory. J Neurosci Res 75:12-24.

Wang H, Storm DR (2003) Calmodulin-regulated adenylyl cyclases: crosstalk and plasticity in the central nervous system. Mol Pharmacol 63:463-468.

Wang H, Pineda VV, Chan GC, Wong ST, Muglia LJ, Storm DR (2003)
Type 8 adenylyl cyclase is targeted to excitatory synapses and required for mossy fiber long-term potentiation. J Neurosci 23:9710-9718.

Wang J, Yeckel MF, Johnston D, Zucker RS (2004) Photolysis of postsynaptic caged $\mathrm{Ca}^{2+}$ can potentiate and depress mossy fiber synaptic responses in rat hippocampal CA3 pyramidal neurons. J Neurophysiol 91:1596-1607.

Wayman GA, Impey S, Wu Z, Kindsvogel W, Prichard L, Storm DR (1994) Synergistic activation of the type I adenylyl cyclase by $\mathrm{Ca}^{2+}$ and Gscoupled receptors in vivo. J Biol Chem 269:25400-25405.

Weisskopf MG, Nicoll RA (1995) Presynaptic changes during mossy fibre LTP revealed by NMDA receptor-mediated synaptic responses. Nature 376:256-259.

Wong ST, Athos J, Figueroa XA, Pineda VV, Schaefer ML, Chavkin CC, Muglia LJ, Storm DR (1999) Calcium-stimulated adenylyl cyclase activity is critical for hippocampus-dependent long-term memory and late phase LTP. Neuron 23:787-798.

Wu ZL, Thomas SA, Villacres EC, Xia Z, Simmons ML, Chavkin C, Palmiter RD, Storm DR (1995) Altered behavior and long-term potentiation in type I adenylyl cyclase mutant mice. Proc Natl Acad Sci USA 92:220-224.

Yang S, Farias M, Kapfhamer D, Tobias J, Grant G, Abel T, Bucan M (2007) Biochemical, molecular and behavioral phenotypes of Rab3a mutations in the mouse. Genes Brain Behav 6:77-96.

Yeckel MF, Kapur A, Johnston D (1999) Multiple forms of LTP in hippocampal CA3 neurons use a common postsynaptic mechanism. Nat Neurosci 2:625-633.

Zalutsky RA, Nicoll RA (1990) Comparison of two forms of long-term potentiation in single hippocampal neurons. Science 248:1619-1624.

Zeng H, Chattarji S, Barbarosie M, Rondi-Reig L, Philpot BD, Miyakawa T, Bear MF, Tonegawa S (2001) Forebrain-specific calcineurin knockout selectively impairs bidirectional synaptic plasticity and working/episodiclike memory. Cell 107:617-629. 